\title{
Overexpression of the hydatidiform mole-related gene F10 inhibits apoptosis in A549 cells through downregulation of BCL2-associated X protein and caspase-3
}

\author{
YALI SONG ${ }^{1}$, GONG ZHANG $^{2}$, XIULAN ZHU $^{1}$, ZHANJUN PANG $^{1}$, FUQI XING $^{1}$ and SONG QUAN $^{1}$ \\ ${ }^{1}$ Center of Reproductive Medicine, Department of Obstetrics and Gynecology, Nanfang Hospital; \\ ${ }^{2}$ Cancer Research Institute, Southern Medical University, Guangzhou 510515, P.R. China
}

Received February 16, 2012; Accepted June 6, 2012

DOI: $10.3892 / \mathrm{ol} .2012 .762$

\begin{abstract}
The aim of this study was to investigate how the overexpression of the hydatidiform mole-related gene F10 affects apoptosis in human lung cancer A549 cells. A549 cells were transfected with pEGFP-N1-F10 (A549-F10) or pEGFP-N1 empty vector (A549-empty). Untransfected A549, A549-F10 or A549-empty cells were examined using the MTT cell proliferation assay and the TUNEL-FITC/Hoechst 33258 apoptosis assay. Western blotting was used to examine the expression levels of the pro-apoptotic genes, BCL2-associated $\mathrm{X}$ protein (BAX) and caspase-3. F10 was stably expressed in A549 cells. From 12 h, A549-F10 cells proliferated markedly faster than the untransfected and A549-empty cells. F10 overexpression also significantly inhibited apoptosis, as shown by the reduced number of TUNEL and Hoechst 33258 double-positive cells. This inhibition was likely due to an F10-induced reduction in the BAX and caspase-3 levels. The results of this study indicate that $\mathrm{F} 10$ overexpression inhibits apoptosis in A549 cells through the downregulation of the pro-apoptotic genes BAX and caspase-3.
\end{abstract}

\section{Introduction}

We recently identified F10, the hydatidiform mole-related gene, using screening of suppression subtractive hybridization cDNA libraries of normal and hydatidiform villi (GenBank accession number, AB196290) (1). Previous studies have suggested that F10 is involved in the malignant transformation of hydatidiform moles, as well as the development of gynecological cancer (2,3). F10 is expressed at low levels in human lung cancer A549 cells. The gene promotes cell proliferation

Correspondence to: Dr Song Quan, Center of Reproductive Medicine, Department of Obstetrics and Gynecology, Nanfang Hospital, Southern Medical University, No. 1838 North Guangzhou Avenue, Guangzhou 510515, P.R. China

E-mail: quansong2008@gmail.com

Key words: F10, A549 cells, apoptosis, BCL2-associated X protein, caspase-3 by upregulating proliferating cell nuclear antigen and cyclin D1 (4), suggesting that F10 plays a pro-proliferative role in accelerating cancer development. Since excessive proliferation and inhibited apoptosis are involved in cancer occurrence and development $(5,6)$, this study aimed to examine whether F10 also exerts anti-apoptotic roles. We induced the overexpression of F10 in A549 cells, examined the apoptosis level and compared the expression of apoptosis-associated genes, including BCL2-associated X protein (BAX) and caspase-3.

\section{Materials and methods}

Cells and reagents. The human lung cancer cell line A549 was maintained in RPMI-1640 medium with $10 \%$ fetal bovine serum in a $37^{\circ} \mathrm{C}, 5 \% \mathrm{CO}_{2}$ incubator. Mouse anti-human BAX and mouse anti-human $\beta$-actin monoclonal antibodies were purchased from Boster Biological Technology, Ltd. (Wuhan, China). Mouse anti-human caspase-3 monoclonal antibody was obtained from Cell Signaling Technology, Inc. (Danvers, MA, USA). Rabbit anti-mouse secondary antibody was purchased from Dako Company (Glostrup, Denmark).

Transfection. F10 was inserted into the pEGFP-N1 vector (Takara Bio, Inc., Shiga, Japan) as an EcoRI-Kpn I fragment and the recombinant plasmid was confirmed by sequencing. The plasmid pEGFP-N1-F10 or pEGFP-N1 empty vector was transfected into A549 cells using lipofectamine 2000 (Invitrogen, Carlsbad, CA, USA). The single clones were selected with G418 (Sigma, St. Louis, MO, USA). The expression of F10 was confirmed by RT-PCR analysis following the manufacturer's instructions (Takara Bio, Inc.). The recombinant cell lines were named A549-F10 and A549-empty, respectively.

MTT cell proliferation assay. Untransfected A549, A549-F10 and A549-empty cells were seeded at $1 \times 10^{4}$ cells/well in 96-well plates in $200 \mu \mathrm{l}$ of medium. The cells were cultured for $0.5,1,6,12,24$ or $48 \mathrm{~h}$ (sextuplicate per time point) before $20 \mu \mathrm{l}$ of $5 \mathrm{mg} / \mathrm{ml}$ MTT (Sigma) was added to each well. Following a 4-h incubation, the cell supernatant was discarded and $150 \mu \mathrm{l} /$ well of DMSO (Sigma) was added. After $5 \mathrm{~min}$ of mixing, the OD value at $490 \mathrm{~nm}$ was measured using a Bio-Tek 
A

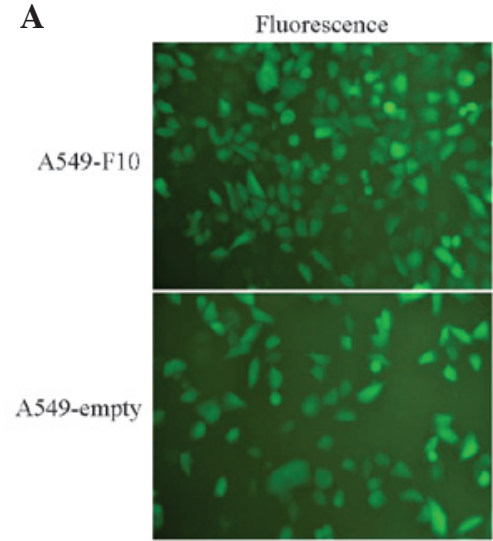

B

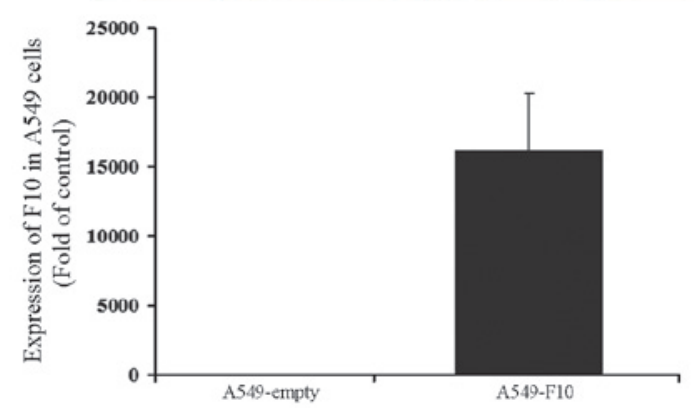

Figure 1. Stable transfection of F10 in A549 cells. (A) A549-F10 and A549-empty cells under a fluorescence microscope (x200). (B) A549-F10 cells stably expressed F10 mRNA. F10 mRNA expression was normalized to GAPDH mRNA expression.

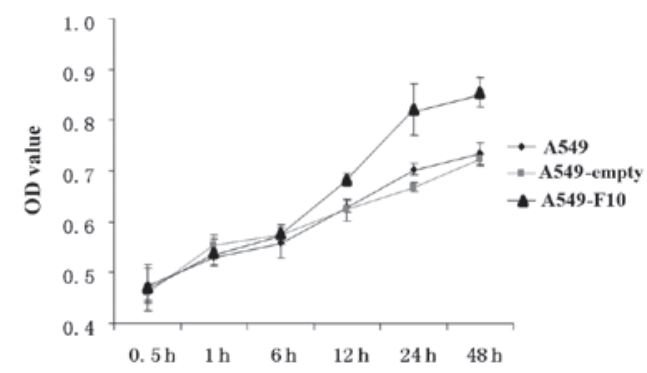

Figure 2. F10 transfection accelerates A549 proliferation. The MTT cell proliferation assay showed that starting from $12 \mathrm{~h}$, A549-F10 cells proliferated markedly faster than A549-empty and untransfected A549 cells ( $\mathrm{n}=6$, $\mathrm{P}<0.05)$. No difference in proliferation was observed between A549-empty and untransfected A549 cells $(\mathrm{P}>0.05)$.

microplate reader (Bio-Rad, Hercules, CA, USA), then the tumor cell growth curve was drawn.

TUNEL-FITC/Hoechst 33258 apoptosis detection assay. Apoptosis was detected using the Annexin V-FITC Apoptosis Detection kit and the Hoechst Staining kit (KeyGEN Biotech., Nanjing, China). Untransfected A549, A549-F10 and A549-empty cells were seeded on coverslips, washed three times in PBS for 5 min each, fixed in $4 \%$ formaldehyde for $20 \mathrm{~min}$ and incubated in $70 \%$ ethanol at $-20^{\circ} \mathrm{C}$ for $30 \mathrm{~min}$. The coverslips were washed a further 3 times and the cells were permeabilized. The permeabilization was performed in $0.1 \%$ Triton $\mathrm{X}-100 / 0.1 \%$ sodium citrate at room temperature for 10 min. After three 5-min washes in PBS, the cells were incubated with $3 \% \mathrm{H}_{2} \mathrm{O}_{2}$ at room temperature for $10 \mathrm{~min}$. After



Figure 3. F10 transfection inhibits apoptosis in A549 cells. (A) Untransfected A549, A549-F10 and A549-empty cells were double-stained with TUNEL-FITC and Hoechst 33258 and observed under a microscope. For each cell type, five fields were randomly selected at x200 magnification and the number of TUNEL-FITC (green) and Hoechst 33258 (blue) double-positive cells was counted. (B) There were markedly fewer apoptotic A549-F10 cells than untransfected A549 and A549-empty cells $(\mathrm{P}<0.001)$. No difference in the apoptotic level was observed between the untransfected A549 and A549-empty cells $(\mathrm{P}>0.05)$.

another three 5-min washes in PBS, the cells were incubated with TdT enzyme at $37^{\circ} \mathrm{C}$ for $90 \mathrm{~min}$, which was protected from light. After two 2-min washes in PBS, the nuclei were stained with Hoechst 33258 at room temperature for $20 \mathrm{~min}$ in the dark. The cells were finally washed in the dark three times in PBS containing 0.5\% Tween 20, 2 min each, and mounted in glycerol. Images were captured using a fluorescence microscope (Nikon, Tokyo, Japan).

Western blot analysis. Lysates from untransfected A549, A549-F10 and A549-empty cells were separated on gels, transferred to membranes, first stained with anti-BAX, anti-caspase- 3 or anti- $\beta$-actin antibody, then stained with HRP-labeled secondary antibodies and developed with an ECL kit. The quantification was analysed using the SensiAnsys software (Shanghai Peiqing Science \& Technology, Co., Ltd., Shanghai, China).

Statistical analysis. Data were analyzed using the SPSS 13.0 software (SPSS, Inc., Chicago, IL, USA) and expressed as mean \pm standard deviation. RT-PCR results were analyzed using the two-sample t-test. For the MTT cell proliferation 
assay, the factorial design analysis of variance (ANOVA) was used to compare inter-group differences. For the TUNEL-FITC/Hoechst 33258 assay and western blot analysis, the results were analyzed by one-way ANOVA followed by Fisher's LSD post hoc tests if variance homogenenity was assumed, or by Welch and Dunnett T3 tests if homogeneity was not assumed. $\mathrm{P}<0.05$ was considered to indicate a statistically significant result.

\section{Results}

Stable transfection of F10 in A549 cells. A549 cells were transfected with pEGFP-N1-F10 or the pEGFP-N1 empty vector and selected for 4 weeks with G418. After another 1-week culture, cells from the two groups were green under a microscope, showing that transfection efficiency was close to $100 \%$ (Fig. 1A). The expression of F10 was confirmed by RT-PCR (A549-F10 vs. A549-empty, $\mathrm{t}=-6.904, \mathrm{P}=0.002$ ) (Fig. 1B).

F10 transfection accelerates A549 proliferation. We compared proliferation among untransfected A549, A549-F10 and A549-empty cells using the MTT assay (Fig. 2). The difference between the groups was significant $(\mathrm{F}=48.039, \mathrm{P}=0.000)$. The effect of time and the correlation between group and time were significant $(\mathrm{F}=323.264, \mathrm{P}=0.000$ and $\mathrm{F}=11.442$, $\mathrm{P}=0.000$, respectively). There was no difference between the three groups at $0,0.5$ and $1 \mathrm{~h}$. After $12 \mathrm{~h}, \mathrm{~A} 549-\mathrm{F} 10$ cells proliferated markedly faster than A549-empty and untransfected A549 cells $(\mathrm{P}<0.05)$. No difference in proliferation was observed between the A549-empty and untransfected A549 cells $(\mathrm{P}>0.05)$.

F10 transfection inhibits apoptosis in A549 cells. To examine the effect of F10 overexpression on apoptosis, untransfected A549, A549-F10 and A549-empty cells were double-stained with TUNEL and Hoechst 33258 (Fig. 3). Apoptotic cells were TUNEL-positive and their nuclei exhibited strong Hoechst blue staining. By contrast, normal cells were TUNEL-negative and showed weak blue nulcei Hoechst staining. TUNEL and Hoechst 33258 double-positive cells were counted and the number of double-positive (apoptotic) cells differed significantly among the three cell lines $(\mathrm{F}=17.472, \mathrm{P}=0.000)$. There were markedly fewer apoptotic A549-F10 cells than untransfected A549 and A549-empty cells ( $\mathrm{P}<0.001)$, suggesting that F10 overexpression inhibits apoptosis in A549 cells. No difference in the apoptotic level was observed between the untransfected A549 cells and A549-empty cells ( $\mathrm{P}=0.816)$.

F10 transfection reduces $B A X$ and caspase-3 protein levels in A549 cells. We next examined how F10 transfection affects the expression of pro-apoptotic genes, BAX and caspase-3 (Fig. 4). Our western blotting results showed significant difference among the three cell lines in the expression of $\mathrm{BAX}($ Welch $=45.966, \mathrm{P}=0.008)$ and caspase- 3 $(\mathrm{F}=268.790, \mathrm{P}=0.000)$. The Dunnett's T3 test demonstrated that A549-F10 cells exhibited lower BAX protein expression than untransfected A549 cells and A549-empty cells $(\mathrm{P}<0.05)$. Similarly, the LSD test revealed that the caspase- 3 expression in A549-F10 cells was markedly lower than that

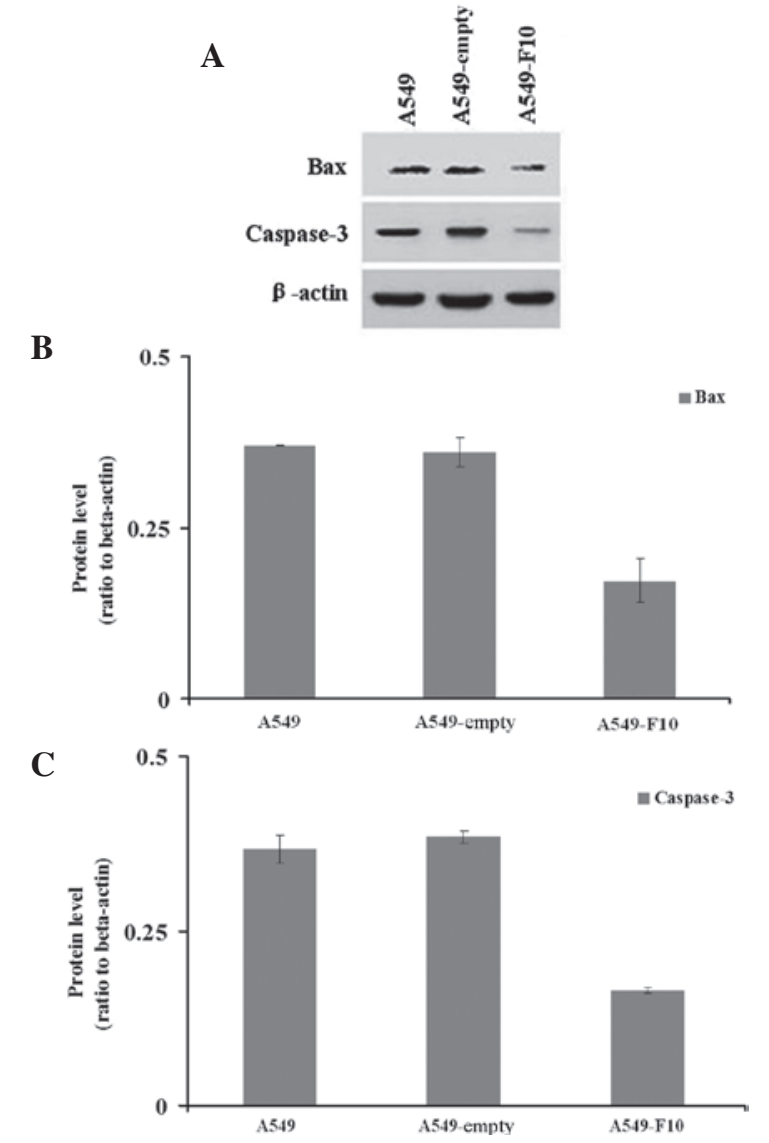

Figure 4. F10 transfection reduces BAX and caspase-3 protein levels in A549 cells. (A) Western blot showing BAX, caspase- 3 and $\beta$-actin (loading control) staining for untransfected A549, A549-empty and A549-F10 cells. Band signals from BAX and caspase- 3 were normalized to those from $\beta$-actin. (B) A549-F10 cells showed lower BAX protein expression than untransfected A549 and A549-empty cells $(\mathrm{P}<0.05)$. No difference was observed between the untransfected A549 and A549-empty cells ( $\mathrm{P}=0.833)$. (C) A549-F10 cells showed lower caspase-3 protein expression than untransfected A549 cells and A549-empty cells $(\mathrm{P}=0.000)$. No difference was observed between the untransfected A549 cells and A549-empty cells $(\mathrm{P}=0.155)$. BAX, BCL2-associated X protein.

in untransfected A549 and A549-empty cells $(\mathrm{P}=0.000)$. No difference in the expression of $\mathrm{BAX}(\mathrm{P}=0.833)$ or caspase- 3 $(\mathrm{P}=0.155)$ was observed between the untransfected A549 and A549-empty cells.

\section{Discussion}

F10 has been suggested to participate in the malignant transformation of hydatidiform moles and the development of the gynecological cancer. Therefore, it is imperative to study the potential role of F10 to improve diagnosis and treatment of cancer. One promising strategy is to establish a cell system overexpressing F10. We previously screened F10 mRNA expression by RT-PCR in eight different cell lines (Bel7402, HIC, HepG2, PC, A549, MGC, 16HBE and 293 cells) (7). The human lung cancer cell line A549 was identified as expressing low levels of F10 and thus served as a model cell system for studies using F10 overexpression. In this study, we transfected A549 cells with pEGFP-N1-F10 plasmid stably and selected single positive clones using G418. F10 mRNA expression was confirmed by RT-PCR. 
The occurrence and development of cancer often involves two aspects: excessive proliferation and inhibited apoptosis (8). F10 has been shown previously to promote tumor cell proliferation (4). In our study this observation was confirmed by the MTT in vitro proliferation assay, which showed that from 12 h, A549 cells overexpressing F10 proliferated markedly faster than untransfected cells or cells transfected with the empty expressing vector. We then examined whether F10 also contributes to cancer development by inhibiting apoptosis. The TUNEL-FITC/Hoechst 33258 staining demonstrated that the level of apoptosis was significantly lower in F10 overexpressing cells.

Apoptosis, first identified in 1972 by Kerr and colleagues, is different from necrosis. It is an actively controlled cell suicide regulated by multiple genes and a series of signal transductions. Pro-apoptotic genes, including caspase-3 and BAX, play important roles in this progress. The caspase family participates in multiple apoptosis-associated physiological and pathological processes (9-11). caspase-3, a member of the caspase family, exerts its pro-apoptotic function through the death receptor (12) and mitochondrial-mediated pathways (13). caspase-3, as an apoptotic effector, may be used as an indicator of apoptosis: upregulation of caspase- 3 expression indicates an increase in apoptosis, whereas downregulation indicates decrease (14). BAX, a pivotal BCL-2 family member, is located in the cytoplasm under normal circumstances. During apoptosis, due to conformational changes, BAX is translocated into the mitochondria to form homodimers, which target and open the mitochondrial intermembrane contact sites and subsequently release cytochrome $\mathrm{C}$ and apoptosis-inducing factors, which in turn promote protein hydrolysis and activate caspases, leading to apoptosis $(15,16)$.

To examine the potential mechanisms underlying the apoptosis inhibition role of F10, we further examined the levels of BAX and caspase- 3 in the presence or absence of F10 overexpression. Western blot analysis demonstrated that F10 overexpression markedly decreases the expression of BAX and caspase- 3 in A549 cells, suggesting that F10 inhibits apoptosis by targeting BAX and caspase- 3 .

In conclusion, our study established a F10-overexpression model in the human lung cancer cell A549 and demonstrated that F10 overexpression promotes cell proliferation and inhibits apoptosis, probably through downregulating the pro-apoptotic genes caspase- 3 and BAX. Since apoptosis involves numerous participants and its mechanism may be different in different cell types, further studies are required to examine the mechanism by which F10 reduces the levels of BAX and caspase-3 and whether other molecules also contribute to the role of F10 in cancer occurrence and development.

\section{Acknowledgements}

The authors would like to thank Yanguo Cui and Xiaomin Cao for their technical assistance. This study was supported by the National Natural Science Foundation of China (no. 30672234).

\section{References}

1. Li GT, Pang ZJ, Zhou J, et al: Cloning of new genes associated with the pathogenesis of hydatidiform mole. Guangdong Medical Journal 27: 22-24, 2006 (In Chinese).

2. Zhou J, Chen SL, Xing FQ, et al: Association of the novel hydatidiform mole-related gene F10 with the invasiveness of trophoblastic tumor. Di Yi Jun Yi Da Xue Xue Bao 25: 171-173, 2005 (In Chinese).

3. Zhou J, Liang W, Li B, et al: The expression of hydatidiform mole associated new gene F10 in different tumor tissues. Guangdong Medical Journal 26: 596-597, 2005 (In Chinese).

4. Cao XM, Pang ZJ, Quan S and Xing FQ: Effect of F10 gene on expression of proliferating cell nuclear antigen and Cyclin D1. Journal of Sun Yat-Sen University (Medical Sciences) 30: 6-9, 2009 (In Chinese).

5. Valásková Z, Kinová S, Danihel L, et al: The complexity of interactions of the tumour growth process. Vnitr Lek 55: 1145-1158, 2009 (In Slovak).

6. Caroppi P, Sinibaldi F, Fiorucci L and Santucci R: Apoptosis and human diseases: mitochondrion damage and lethal role of released cytochrome $\mathrm{C}$ as proapoptotic protein. Curr Med Chem 16: 4058-4065, 2009.

7. Cao XM, Pang ZJ and Quan S: Construction and identification of a stable eukaryotic expression system for F10 gene. Nan Fang Yi Ke Da Xue Xue Bao 28: 57-59, 2008 (In Chinese).

8. Sarcević B: Apoptosis in tumors. Acta Med Croatica 63 (Suppl 2): 43-47, 2009 (In Croatian).

9. Kumar S and Dorstyn L: Analysing caspase activation and caspase activity in apoptotic cells. Methods Mol Biol 559: 3-17, 2009.

10. Vaculova A and Zhivotovsky B: Caspases: determination of their activities in apoptotic cells. Methods Enzymol 442: 157-181, 2008.

11. Denault JB and Salvesen GS: Apoptotic caspase activation and activity. Methods Mol Biol 414: 191-220, 2008.

12. Wang Y, Sun LG and Xia CH: Caspase-mediated Fas apoptosis pathway. World Chinese Journal of Digestology 14: 3439-3442, 2006 (In Chinese).

13. Jin LF and Chen TY: Proteinum family of Bcl-2 gene and apoptosis. Med Recapitul 11: 446-447, 2005 (In Chinese).

14. Mazumder S, Plesca D and Almasan A: Caspase-3 activation is a critical determinant of genotoxic stress-induced apoptosis. Methods Mol Biol 414: 13-21, 2008.

15. Pan G, O'Rourke K and Dixit VM: Caspase-9, Bcl-XL, and Apaf-1 form a ternary complex. J Biol Chem 273: 5841-5845, 1998.

16. Li P, Nijhawan D, Budihardjo I, et al: Cytochrome c and dATPdependent formation of Apaf-1/caspase- 9 complex initiates an apoptotic protease cascade. Cell 91: 479-489, 1997. 